\title{
Ultrafast optical control of magnetization dynamics in polycrystalline bismuth doped iron garnet thin films
}

Marwan Deb, Mircea Vomir, Jean-Luc Rehspringer, and Jean-Yves Bigot

Citation: Appl. Phys. Lett. 107, 252404 (2015); doi: 10.1063/1.4938538

View online: https://doi.org/10.1063/1.4938538

View Table of Contents: http://aip.scitation.org/toc/apl/107/25

Published by the American Institute of Physics

\section{Articles you may be interested in}

Perspective: Ultrafast magnetism and $\mathrm{THz}$ spintronics

Journal of Applied Physics 120, 140901 (2016); 10.1063/1.4958846

Experimental demonstration of 55 -fs spin canting in photoexcited iron nanoarrays

Applied Physics Letters 110, 082404 (2017); 10.1063/1.4977097

Non-thermal optical excitation of terahertz-spin precession in a magneto-optical insulator Applied Physics Letters 108, 032404 (2016); 10.1063/1.4940241

Spin-photo-currents generated by femtosecond laser pulses in a ferrimagnetic $\mathrm{GdFeCo} / \mathrm{Pt}$ bilayer Applied Physics Letters 110, 072402 (2017); 10.1063/1.4976202

Temperature-dependent Faraday rotation and magnetization reorientation in cerium-substituted yttrium iron garnet thin films

APL Materials 5, 036104 (2017); 10.1063/1.4976817

Synthesis and characterization of yttrium iron garnet (YIG) nanoparticles - Microwave material AIP Advances 7, 056405 (2017); 10.1063/1.4973199

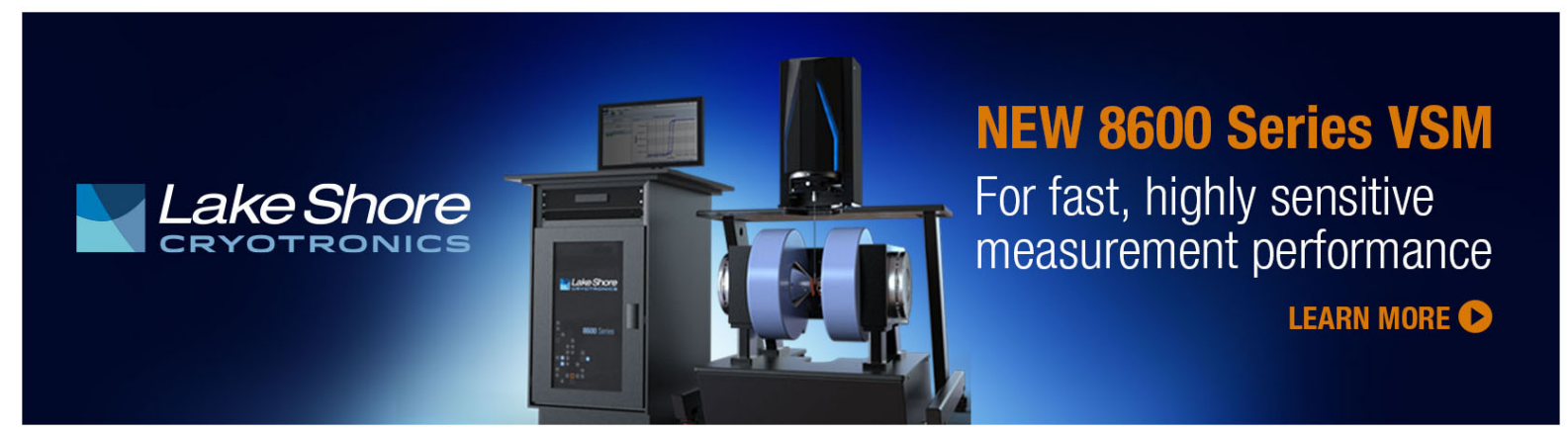




\title{
Ultrafast optical control of magnetization dynamics in polycrystalline bismuth doped iron garnet thin films
}

\author{
Marwan Deb, ${ }^{\text {a) }}$ Mircea Vomir, Jean-Luc Rehspringer, and Jean-Yves Bigot \\ Institut de Physique et Chimie des Matériaux de Strasbourg, UMR 7504, CNRS, Université de Strasbourg, \\ BP 43, 23 rue du Loess, 67034 Strasbourg Cedex 02, France
}

(Received 24 October 2015; accepted 11 December 2015; published online 24 December 2015)

\begin{abstract}
Controlling the magnetization dynamics on the femtosecond timescale is of fundamental importance for integrated opto-spintronic devices. For industrial perspectives, it requires to develop simple growth techniques for obtaining large area magneto-optical materials having a high amplitude ultrafast Faraday or Kerr response. Here we report on optical pump probe studies of light induced spin dynamics in high quality bismuth doped iron garnet polycrystalline film prepared by the spin coating method. We demonstrate an ultrafast non-thermal optical control of the spin dynamics using both circularly and linearly polarized pulses. (C) 2015 AIP Publishing LLC.

[http://dx.doi.org/10.1063/1.4938538]
\end{abstract}

The ultrafast manipulation and switching of the magnetization using femtosecond laser pulses is a topic of great interest for magnetic storage and spintronic technologies. ${ }^{1}$ For recording application purposes, several directions are undertaken. On one hand, it is envisaged to use thermally induced changes of the magnetization using femtosecond pulses, as initially proposed in ferromagnetic metallic nickel films. ${ }^{2}$ This is of particular interest in the context of heat assisted magnetic recording ${ }^{3}$ that takes advantage of large anisotropy materials such as FePt nanoparticles. ${ }^{4}$ On the other hand, a direct control of the magnetization with the polarization of the light pulses is feasible, as initially demonstrated in metallic ferrimagnetic films such as $\mathrm{GdFeCo}$ alloys ${ }^{5}$ and extended recently to multilayered ferromagnetic films. ${ }^{6}$ When recording is not the primary goal, one can also take advantage of the pulse polarization to induce coherent processes. ${ }^{7}$ With that respect, transparent dielectric magnetic materials such as Garnets are well suited as one can either control optically the precession of the magnetic vector ${ }^{8}$ or to generate magneto-optical fourwave mixing signals. ${ }^{9}$ All these approaches and the corresponding methods of investigation of the spin dynamics are very promising once implemented at the nanoscale. ${ }^{10}$ For obvious reasons, one has in addition to envisage large scale material surfaces as well as easy fabrication techniques. For metallic films and nanoparticles, sputtering techniques are well appropriate. For dielectrics, the situation is more complex as it is difficult to obtain large-area polycrystalline thin garnet films with high Faraday magneto-optical responses.

In this letter we report an approach both from the optical and material viewpoints. On one side, we have made $\mathrm{Bi}$ :doped iron garnets with a spin coating technique, versatile enough to realize wide range of garnets with areas of several inches. On the other side, we demonstrate that the control can be done by both circularly and linearly polarized pulse, which offer more freedom for manipulating the magnetic moments. We forecast that our results will find a variety of applications in integrated opto-spintronic devices as they

\footnotetext{
${ }^{\text {a) }}$ Author to whom correspondence should be addressed. Electronic mail: marwan.deb@ipcms.unistra.fr
}

have an ultrafast response time and can be prepared by a simple and low-cost method. An important feature is that the poly-crystallinity does not affect significantly the observed dynamics because it is mostly related to a non-thermal opto-magnetic excitation. In such situation, the magnetocrystalline anisotropy is not modified as compared to thermally induced effects.

Let us remind some fundamentals of the nonlinear magneto-optical response that will be useful hereafter. Nonthermal opto-magnetic excitation mechanisms have recently allowed an efficient control of the magnetization dynamics in ferromagnetic and antiferromagnetic dielectric material. The inverse Faraday effect (IFE) is one of the most important. It has been demonstrated in a number of mono- and singlecrystalline dielectric materials such as $(\mathrm{LuYbi})_{3}[\mathrm{FeGA}]_{5} \mathrm{O}_{12}$, ${ }^{11}$ $\mathrm{DyFeO}_{3},{ }^{12} \mathrm{ErFeO}_{3}{ }^{13}$ and $\mathrm{NiO} .{ }^{14}$ The IFE shows up experimentally by a $180^{\circ}$ phase shift in the spin precession when the helicity of the circularly polarized light is reversed. A nonthermal control of the spin precession is also observed with linearly polarized light in signal-crystalline dielectric materials such as $\mathrm{FeBO}_{3},{ }^{15} \mathrm{DyFeO}_{3}{ }^{16}$ and $\mathrm{Gd}_{4 / 3} \mathrm{Yb}_{2 / 3} \mathrm{BiFe}_{5} \mathrm{O}_{12}$. ${ }^{17}$ The excitation mechanism has been attributed to a photon induced magnetic anisotropy (PIA) and/or the inverse Cotton-Mouton effect (ICME). Non-thermal processes are interesting to investigate as they could in principle be used to switch the magnetization in appropriately designed material. ${ }^{5,18}$ However, in most published results, this process has been demonstrated in mono- and single-crystalline films which fabrication is complex and costly if industrial purposes are envisaged.

The bismuth-doped iron garnet (BIG) materials have very large magneto-optical rotation and ellipticity $\left(\sim 10^{4} \mathrm{deg} /\right.$ $\mathrm{cm}$ at $2.4 \mathrm{eV}$ ), with a relatively high Curie temperature ( $\sim 660 \mathrm{~K})$ and have a low absorption coefficient in the visible and infrared spectrum $(\mathrm{gap} \sim 2.5 \mathrm{eV}) .{ }^{19}$ They are therefore well adapted for non-reciprocal magneto-optical devices. ${ }^{20}$

The sample studied in the present work is a $200 \mathrm{~nm}-$ thick polycrystalline $\mathrm{Bi}_{0.3} \mathrm{Y}_{2.7} \mathrm{Fe}_{5} \mathrm{O}_{12}$ garnet film prepared by a spin coating method into a glass substrate. ${ }^{21}$ This method has the considerable advantage of being easy to implement, cheap, and allows producing large area samples. 
The measurements of the static MO properties were performed using a custom magnetometer setup based on $90^{\circ}$ modulation-polarization technique. The white light emitted by a $150 \mathrm{Xe}$ arc lamp is polarized by a Rochon polarizer and modulated at a frequency of $50 \mathrm{kHz}$ by a Hinds photo-elastic modulator (PEM). The modulated light is focused onto the magnetic sample at nearly normal incidence. The transmitted light is collimated and analyzed by a Rochon polarizer. The analyzed light is focused into a monochromator equipped with a motorized filter wheel to eliminate higher-order diffraction and three diffraction gratings to cover the 200-2200 nm spectral range. The synchronous detection consists of a photomultiplier and two lock-in amplifiers at the fundamental and double PEM frequencies. The first harmonic signal is proportional to the ellipticity $\varepsilon_{\mathrm{F}}$, whereas the second harmonic one is proportional to the rotation $\Theta_{\mathrm{F}}$. Fig. 1(a) shows normalized polar (field perpendicular) and longitudinal (fields in plane) Faraday hysteresis loops of $\mathrm{Bi}_{0.3} \mathrm{Y}_{2.7} \mathrm{Fe}_{5} \mathrm{O}_{12}$. The perpendicular saturation field $(\sim 2.1 \mathrm{kG})$ is about three times larger than the in-plane saturation field $(\sim 0.75 \mathrm{kG})$. The normalized remanence $\left(\mathrm{M}_{\mathrm{r}} / \mathrm{M}_{\mathrm{s}}\right)$ is 0.6 and 0.1 for the perpendicular and in-plane configurations, respectively. These results clearly show that the easy axis of the magnetization is parallel to the surface of the film. The spectral dependency of the Faraday and Kerr rotations $\left(\Theta_{\mathrm{F}}, \Theta_{\mathrm{K}}\right)$ and ellipticities $\left(\varepsilon_{\mathrm{F}}, \varepsilon_{\mathrm{K}}\right)$, measured in the polar configuration with a saturation external field, is shown in Figures 1(b) and 1(c). $\Theta_{\mathrm{F}}$ is negative above $475 \mathrm{~nm}$ and positive between 475 and $335 \mathrm{~nm}$ with a maximum at $400 \mathrm{~nm}$, whereas $\varepsilon_{\mathrm{F}}$ has two peaks centered at 470 and $355 \mathrm{~nm}$. On the other hand, the MO Kerr signal is large below the optical band gap, i.e., for wavelengths larger than $475 \mathrm{~nm}$ : $\Theta_{\mathrm{k}}$ reaches a value of $1.5^{\circ}$ near $500 \mathrm{~nm}$ and $\varepsilon_{\mathrm{k}}$ reaches a value of $1^{\circ}$ at 515 and $455 \mathrm{~nm}$. Above the band gap, the highest MO response is $0.58^{\circ}$. The large Kerr signal below the band gap is due to multiple reflections in the film that show up in the MO Kerr response. Let us recall that the corresponding optical transitions are well explained, taking into account the crystal field energy level of Bi doped iron garnet. ${ }^{22,23}$

All measured MO spectra of our polycrystalline film are in good agreement with previous MO studies of monocrystalline bismuth doped yttrium iron garnet. ${ }^{19,23,24}$ These values of the MO signal can also be compared with the ones prepared using metal organic decomposition. ${ }^{25}$ We find that for the same film thickness and concentration $\mathrm{X}_{\mathrm{Bi}}$ of bismuth, our MO signal at the wavelength $633 \mathrm{~nm}$ is twice as large as the ones made by MOD. The procedure we adopted is to consider the linear variation of $\Theta_{\mathrm{F}}$ with $\mathrm{X}_{\mathrm{Bi}}$ as reported in Ref. 19. In addition to these static MO measurements, the $\mathrm{X}$ ray characterization confirms the high quality of the film.

The dynamical properties were measured at room temperature in the magneto-optical pump-probe configuration sketched in Fig. 2. Briefly, 120 fs laser pulses with a repetition rate of $1 \mathrm{kHz}$ are used to generate the pump and probe beams. The pump beam $(800 \mathrm{~nm})$ excites the sample at a small angle from the normal to the sample $\left(\sim 6^{\circ}\right)$, while the probe beam $(400 \mathrm{~nm})$ is perpendicular to the film plane. The spot size of the pump was $100 \mu \mathrm{m}$, while the probe spot was $30 \mu \mathrm{m}$. The polarization of the probe beam is linear, whereas the pump beam polarization can be varied using a half
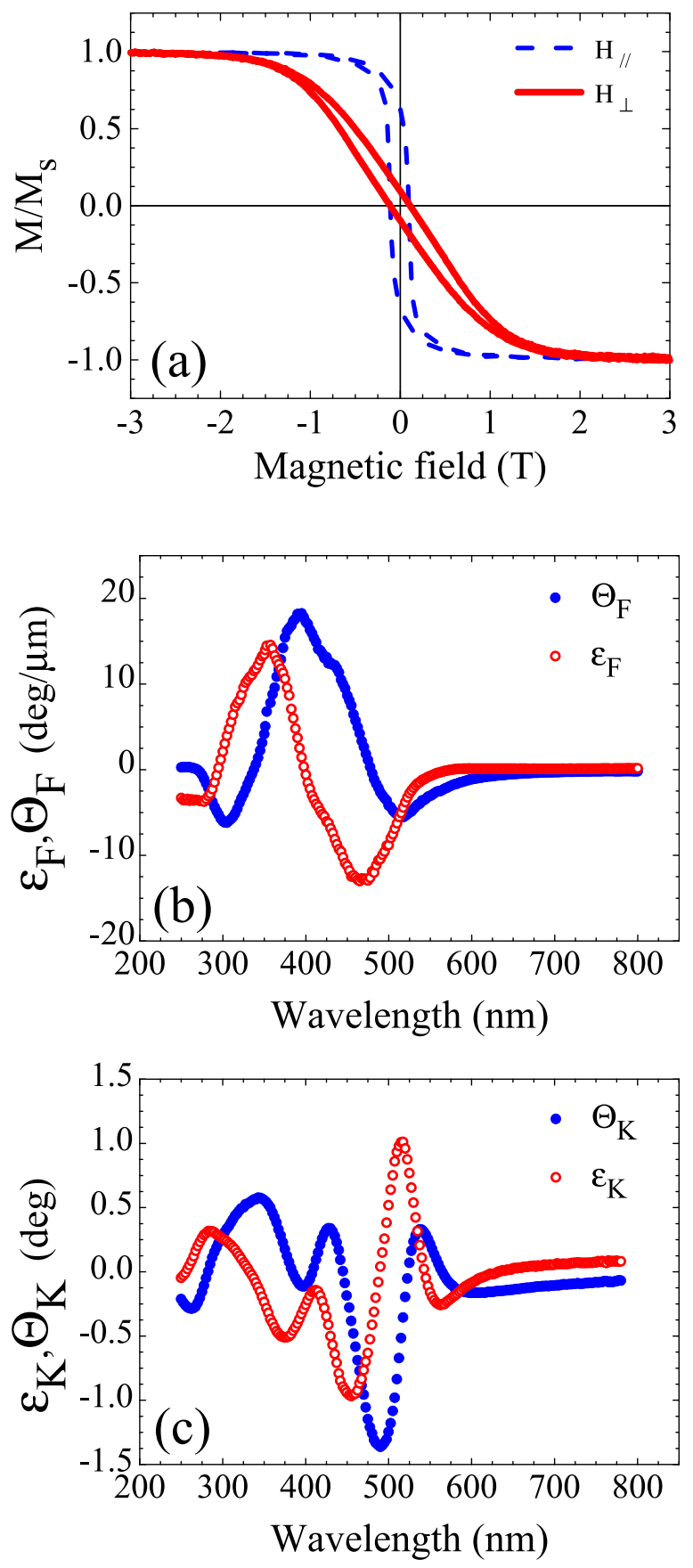

FIG. 1. Static room temperature magneto-optical properties of polycrystalline $\mathrm{Bi}_{0.3} \mathrm{Y}_{2.7} \mathrm{Fe}_{5} \mathrm{O}_{12}$ thin film. (a) Normalized hysteresis loops measured in polar (solid line) and longitudinal (dashed line) configuration. (b) and (c) Magneto-optical polar Faraday (b) and Kerr (c) spectra. The filled and opened symbols represent, respectively, the magneto-optical rotation $\left(\Theta_{\mathrm{F}}\right.$, $\left.\Theta_{\mathrm{K}}\right)$ and ellipticity $\left(\varepsilon_{\mathrm{F}}, \varepsilon_{\mathrm{K}}\right)$.

wave-plate (HWP) and a quarter wave-plate (QWP). All pump-induced time resolved Faraday rotation signals are fitted with the damped sine function

$$
\Theta_{F}(t)=\Theta_{F 0}+\Theta_{F o s c} e^{-\frac{t}{\tau}} \sin (2 \pi f t-\phi),
$$

where $\Theta_{\text {Fosc }}$ is the initial amplitude of oscillation, $f$ is the frequency, $\frac{1}{\tau}$ is the relaxation rate, $\phi$ is the initial phase of oscillation, and $\Theta_{F 0}$ is a magneto-optical offset.

The time resolved Faraday rotation induced by right $(\sigma+)$ and left $(\sigma-)$ circularly polarized pump beam for an 

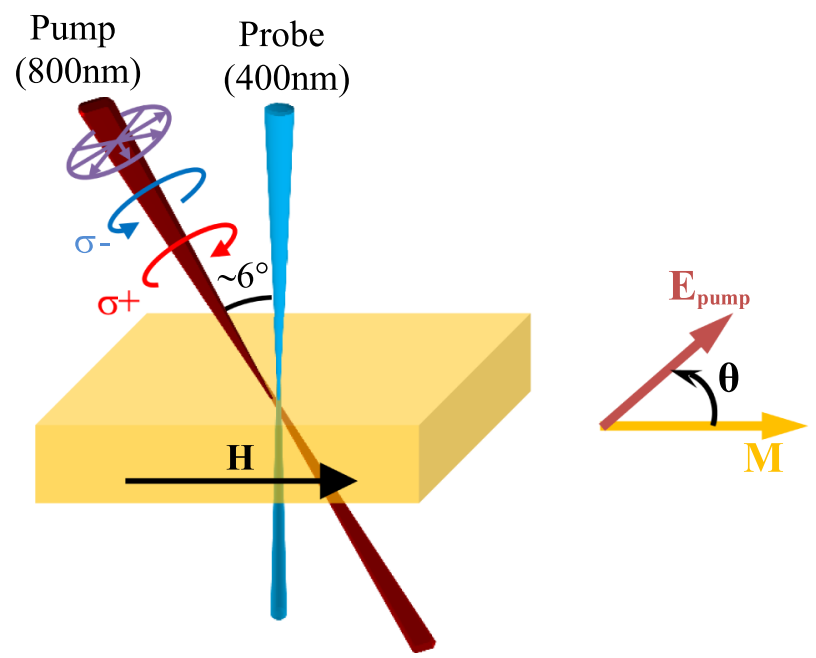

FIG. 2. Sketch of the experimental time resolved MO configuration.

external magnetic field of $3.6 \mathrm{kG}$ is shown in Figure 3, showing oscillations with a $\pi$ dephasing for opposite pump helicities. This result can be explained by the inverse Faraday effect, where a magnetic field is generated by circular polarized light in the direction of the propagating pump beam. This field has opposite sign for $\sigma+$ and $\sigma-$ which results in a $180^{\circ}$ phase shift for the spin precession. The inset of Figure 2 displays the variation of the amplitude as a function of the fast axis of the QWP relative to the s-polarized pump beam. The amplitude follows a twofold sinusoidal function $(\sin 2 \theta)$. It is maximum for circular polarization $(\theta= \pm 45)$ and very weak close to the $\mathrm{p}$ - or s-polarized pump $(\theta=0$ and \pm 90$)$.

We have also studied the precession of the magnetization as a function of the external magnetic field. The dependence of the time resolved Faraday rotation induced by left $(\sigma-)$ circular pump beam with magnetic field strength is shown in Figure 4(a). To quantify the various changes, each time resolved Faraday rotation is fitted with Eq. (1). The precession frequencies as shown in Fig. 4(a) are in the range of a few gigahertz, which is in good argument with previous

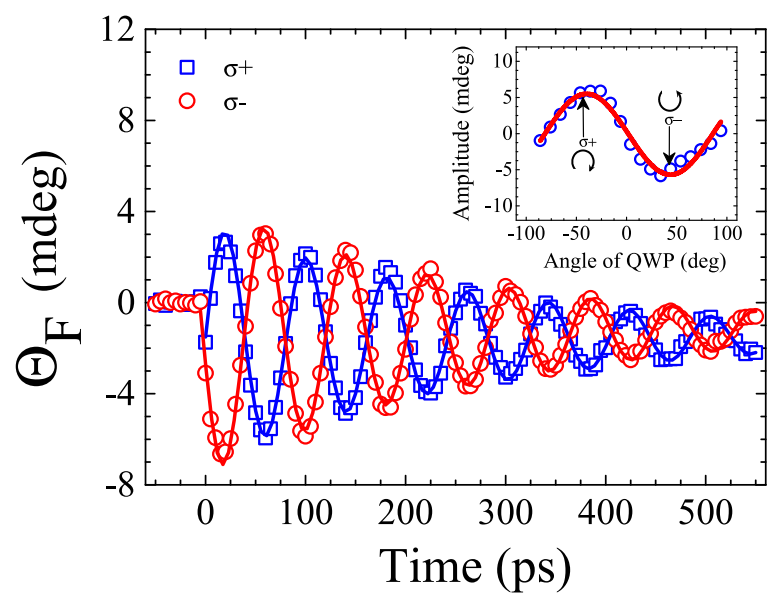

FIG. 3. Time resolved Faraday rotation induced by right $(\sigma+$, opened square) and left ( $\sigma-$, opened circle) circular polarization pump beam. The magnetization precession has opposite phases for the two helicities $\sigma+$ and $\sigma-$. Inset: oscillations amplitude as a function of the orientation of the quarter wave plate. The maxima of oscillations amplitude are for circular polarization and oscillations, while very small amplitude is measured for linear s-polarization. The solid line in inset is the guide to the eye.
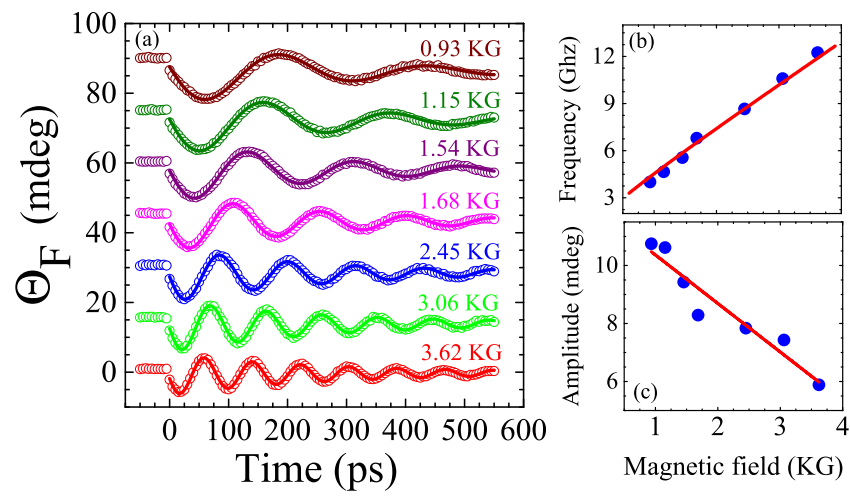

FIG. 4. (a) Time resolved Faraday rotation induced by left $(\sigma-)$ circular pump beam as a function of external magnetic field. For better visualization, a vertical offset has been introduced. (b) and (c) Variation of the precession frequency and oscillations amplitude as a function of the $\mathrm{H}_{\mathrm{ext}}$. The solid line in (b) is a fit with the Kittel formula.

ferromagnetic resonance (FMR) results in Bi-YIG. ${ }^{26}$ The variation of the precession frequency can be described by the Kittel formula adapted to the case of a thin film and to our experimental configuration ${ }^{27}$

$$
\omega=\gamma \sqrt{H_{e x t}\left(H_{e x t}+H_{e f f}\right)},
$$

where $\omega$ is the angular precession frequency, $\gamma$ the gyromagnetic ratio, $\mathrm{H}_{\mathrm{ext}}$ the external magnetic field, and the effective field $\mathrm{H}_{\text {eff }}$ is defined as $\left(4 \pi \mathrm{M}_{\mathrm{s}}-\mathrm{H}_{\mathrm{K}}\right)$ where $\mathrm{H}_{\mathrm{K}}$ is the uniaxial anisotropy. The adjustment of Fig. 4(a) with Eq. (2) yields: $\mathrm{H}_{\text {eff }}=1.46 \mathrm{kG}$, using $\gamma=2.8 \mathrm{GHz} / \mathrm{kG}$. The result is shown in Fig. 4(b).

In Figure 4(c), we show that the amplitude of precession decreases with increasing field $\mathrm{H}_{\text {ext }}$. This is in agreement with the reduction of the canting angle induced by the IFE between the magnetization and the external magnetic field for larger field values.

To investigate further the excitation of the spin dynamics in polycrystalline garnet films, we measured the time resolved Faraday rotation obtained for a linear pump polarization with different angle $\theta$ as shown in Figure 5(a). The linear polarized pump pulses excite the same mode of magnetization precession as in the case of a circularly polarized
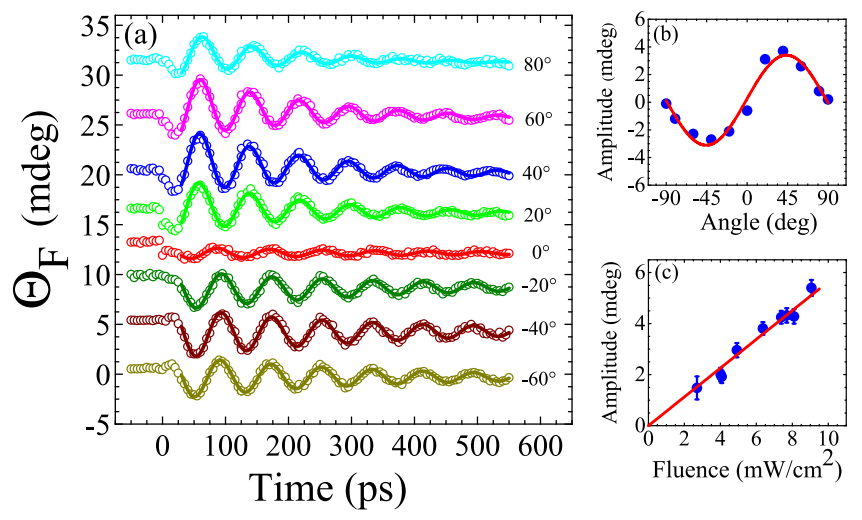

FIG. 5. (a) Time resolved Faraday rotation measured for an external magnetic field of $3.6 \mathrm{kG}$ for different linear pump polarization angles $\theta$. For better visualization, a vertical offset has been introduced. (b) and (c) Oscillations amplitude as a function of the linear pump polarization angle and the energy density of pump pulses. The solid line in (c) is a linear fit. 
pump pulses. In addition, the amplitude and phase of the precession change with the linear polarization angle $\theta$. Indeed, the spin precession vanishes for parallel or perpendicular orientation of the pump with respect to the magnetic field, whereas its absolute amplitude is maximum for $\theta= \pm 45$ (see Figure 5(b)). The negative sign in the initial amplitude of precession reveals the precession with opposite phase. From Figure 3(c), we show that the initial amplitude of precession depends linearly on the energy density of the pump pulses.

As mentioned above, the so-called PIA and ICME are the two main known mechanisms for the non-thermal optical control of spin precession with the linear polarized light. ${ }^{28}$ These two mechanisms depend on the polarization angle and have been observed in the case of signal-crystalline garnet thick films. ${ }^{11,17}$ More recently, in addition to the PIA, an excitation of the spin dynamics with small amplitude via a polarization independent impulsive effect has been observed in $\mathrm{Bi}_{3} \mathrm{Fe}_{5} \mathrm{O}_{12}$ single-crystalline films. ${ }^{29}$

Let us mention that in Figure 5(a), there is a slight variation of the initial phase of precession for a polarization $\theta=0^{\circ}$ and also for $90^{\circ}$ (not shown in the graph). It is a weak effect as can be deduced by adding the amplitudes of the precession signals $\operatorname{Sig}+=(\operatorname{Sig}(\theta)+\operatorname{Sig}(\theta+\pi / 2)) / 2$. This phenomenon is the same as reported in Ref. 29 as it has the same phase and does not depend on the polarization of light. In addition, since in our case the precession amplitude induced by linearly polarized light increases with decreasing magnetic field, and the phase is $\sim 0[\bmod \pi]$, also as reported in Ref. 29, we conclude that the PIA is the dominant process.

In summary, we have investigated the laser induced magnetization dynamics in bismuth doped iron garnet polycrystalline film with a femtosecond magneto-optical pump-probe technique. The static magneto-optical spectral measurements show a large amplitude magneto-optical response for our thin film prepared by a spin coating method. For the pump probe experiments, a non-thermal excitation and control of magnetization dynamics have been demonstrated by both circular and linear polarized pump pulses.

The authors acknowledge the financial support of the Agence Nationale de la Recherche in France via the project EQUIPEX UNION: \# ANR-10-EQPX-52.

${ }^{1}$ J.-V. Bigot, W. Hübner, T. Rasing, and R. Chantrell, Ultrafast Magnetism I (Springer International Publishing, 2015).
${ }^{2}$ E. Beaurepaire, J. C. Merle, A. Daunois, and J. Y. Bigot, Phys. Rev. Lett. 76(22), 4250 (1996)

${ }^{3}$ Y. J. Chen, H. Z. Yang, S. H. Leong, B. Santoso, J. Z. Shi, B. X. Xu, and J. W. H. Tsai, J. Appl. Phys. 117(17), 17C106 (2015).

${ }^{4}$ J.-V. Bigot, J. Kim, M. Vomir, O. Mosendz, S. Jain, and D. Weller, in International Workshop on Nanomaterials, Nancy, France, 2014; O. Mosendz, S. Pisana, J. W. Reiner, B. Stipe, and D. Weller, J. Appl. Phys. 111(7), 07B729 (2012).

${ }^{5}$ C. D. Stanciu, F. Hansteen, A. V. Kimel, A. Kirilyuk, A. Tsukamoto, A. Itoh, and Th. Rasing, Phys. Rev. Lett. 99(4), 047601 (2007).

${ }^{6}$ S. Mangin, M. Gottwald, C. H. Lambert, D. Steil, V. Uhliŕ, L. Pang, M. Hehn, S. Alebrand, M. Cinchetti, G. Malinowski, Y. Fainman, M. Aeschlimann, and E. E. Fullerton, Nat. Mater. 13(3), 286 (2014).

${ }^{7}$ J.-Y. Bigot, M. Vomir, and E. Beaurepaire, Nat. Phys. 5(7), 515 (2009).

${ }^{8}$ F. Hansteen, A. Kimel, A. Kirilyuk, and T. Rasing, Phys. Rev. Lett. 95(4), 047402 (2005).

${ }^{9}$ M. Barthelemy, M. Vomir, M. S. Piaia, H. Vonesch, P. Molho, B. Barbara, and J.-Y. Bigot, e-print arXiv:1311.0080.

${ }^{10}$ J.-Y. Bigot, Nat. Mater. 12(4), 283 (2013); J.-Y. Bigot and M. Vomir, Ann. Phys. 525(1-2), 2 (2013).

${ }^{11}$ F. Hansteen, A. Kimel, A. Kirilyuk, and T. Rasing, Phys. Rev. B 73(1), 014421 (2006).

${ }^{12}$ A. V. Kimel, A. Kirilyuk, P. A. Usachev, R. V. Pisarev, A. M. Balbashov, and T. Rasing, Nature 435(7042), 655 (2005).

${ }^{13}$ J. A. de Jong, A. V. Kimel, R. V. Pisarev, A. Kirilyuk, and T. Rasing, Phys. Rev. B 84(10), 104421 (2011).

${ }^{14}$ T. Satoh, S. J. Cho, R. Iida, T. Shimura, K. Kuroda, H. Ueda, Y. Ueda, B. A. Ivanov, F. Nori, and M. Fiebig, Phys. Rev. Lett. 105(7), 077402 (2010).

${ }^{15}$ A. M. Kalashnikova, A. V. Kimel, R. V. Pisarev, V. N. Gridnev, A. Kirilyuk, and T. Rasing, Phys. Rev. Lett. 99(16), 167205 (2007).

${ }^{16}$ R. Iida, T. Satoh, T. Shimura, K. Kuroda, B. A. Ivanov, Y. Tokunaga, and Y. Tokura, Phys. Rev. B 84(6), 064402 (2011).

${ }^{17}$ I. Yoshimine, T. Satoh, R. Iida, A. Stupakiewicz, A. Maziewski, and T. Shimura, J. Appl. Phys. 116(4), 043907 (2014).

${ }^{18}$ C-H. Lambert, S. Mangin, B. S. D. Ch. S. Varaprasad, Y. K. Takahashi, M. Hehn, M. Cinchetti, G. Malinowski, K. Hono, Y. Fainman, M. Aeschlimann, and E. E. Fullerton, Science 345(6202), 1337 (2014).

${ }^{19}$ P. Hansen and J. P. Krumme, Thin Solid Films 114(1-2), 69 (1984).

${ }^{20} \mathrm{G}$. Winkler, Magnetic Garnets/Gerhard Winkler (Vieweg, Braunschweig, 1981).

${ }^{21}$ J. L. Rehspringer, J. Bursik, D. Niznansky, and A. Klarikova, J. Magn. Magn. Mater. 211(1-3), 291 (2000).

${ }^{22}$ G. F. Dionne and G. A. Allen, J. Appl. Phys. 75(10), 6372 (1994).

${ }^{23}$ M. Deb, E. Popova, A. Fouchet, and N. Keller, J. Phys. D: Appl. Phys. 45(45), 455001 (2012).

${ }^{24}$ S. Wittekoek, T. J. A. Popma, J. M. Robertson, and P. F. Bongers, Phys. Rev. B 12(7), 2777 (1975).

${ }^{25}$ G. Lou, T. Yoshida, and T. Ishibashi, J. Appl. Phys. 117(17), 17A749 (2015).

${ }^{26}$ E. Popova, A. F. F. Galeano, M. Deb, B. Warot-Fonrose, H. Kachkachi, F. Gendron, F. Ott, B. Berini, and N. Keller, J. Magn. Magn. Mater. 335, 139 (2013).

${ }^{27}$ C. Kittel, Phys. Rev. 73(2), 155 (1948).

${ }^{28}$ A. Kirilyuk, A. V. Kimel, and T. Rasing, Rev. Mod. Phys. 82(3), 2731 (2010).

${ }^{29}$ B. Koene, M. Deb, E. Popova, N. Keller, T. Rasing, and A. Kirilyuk, Phys. Rev. B 91(18), 184415 (2015). 